\title{
ADDITIONS TO THE BIRDS OF BILIGIRI RANGASWAMY TEMPLE WILDLIFE SANCTUARY, WESTERN GHATS, INDIA
}

\author{
N.A. Aravind ${ }^{1}$, Dinesh Rao $^{2}$ and P.S. Madhusudan ${ }^{3}$ \\ ${ }^{1,2,3}$ Ashoka Trust for Research in Ecology and the Environment, \#659, 5' 'A' Main, Hebbal, Bangalore, Karnataka 560024, India. \\ Email: ${ }^{1}$ aravind@atree.org, ${ }^{2}$ dinesh@atree.org \\ ${ }_{3}^{3}$ Present address: Salim Ali School of Ecology, Pondicherry University, Kalapet, Pondicherry 605004, India.
}

\begin{abstract}
Biligiri Rangaswamy Temple Wildlife Sanctuary (BRT WLS) is located in the middle of the bridge between Eastern and Western Ghats. There are about 245 birds in the Sanctuary. The present study was aimed to determine birds in various macrohabitats in BRT WLS. Birds were monitored and censused during different seasons for 18 months using transects that were laid in different vegetation types. Twenty-eight species were newly recorded in the Sanctuary out of which 15 are water birds.
\end{abstract}

\section{Keywords \\ Biligiri Rangaswamy Temple Wildlife Sanctuary, birds, checklist}

\section{Introduction}

Biligiri Rangaswamy Temple Wildlife Sanctuary (BRTWLS) $\left(77^{0}-\right.$ $77^{\circ} 16^{\prime} \mathrm{E}$ and $11^{\circ} 47^{\prime}-12^{\circ} 9^{\prime} \mathrm{N}$ ) gets the name Biligiri from the white cliff on which the Temple of Lord Rangaswamy is situated. The Sanctuary is located to the east of the Western Ghats in Karnataka State. It was declared a Wildlife Sanctuary in 1987 and it covers an area of about $540 \mathrm{~km}^{2}$.

Altitude ranges from 600 to $1800 \mathrm{~m}$. Temperature ranges from $10^{\circ}$ to $25^{\circ} \mathrm{C}$ and rainfall is around $600 \mathrm{~mm}$ at the base and $3000 \mathrm{~mm}$ at hilltop per year. This wide range of climatic conditions along with the altitude variations within the small area of the Sanctuary have translated it into a highly heterogeneous forest vegetation types - scrub, deciduous, riparian, evergreen, sholas and grasslands.

The BRTWLS is inhabited by indigenous people called Soligas. These tribes were hunter-gatherers and used to practice shifting cultivation. Although the area was declared a Wildlife Sanctuary, collection of non-timber forest products (NTFP) is still carried out.
BRTWLS is very rich in flora and fauna. There are about 1200 species of angiosperms (Ganesan \& Vanaraj, unpublished) and 245 species of birds. Apart from these, this area harbours 150 species of butterflies (Aravind \& Dinesh, in prep.) and 35 species of mammals, which includes endangered species like the Indian Tiger (Panthera tigris), Asian Elephant (Elephas maximus), Four-horned Antelope (Tetraceros quadricornis), etc.

The aim of our study was to ascertain the nature of bird assemblages in various macrohabitat types in and around BRTWLS, which is an offshoot of the main Western Ghats and is the eastern most extension of the Ghats. This unique extension of Western Ghats constitutes a live bridge between the Eastern Ghats and Western Ghats with the BRTWLS located almost in the middle of this bridge. Thus the biota of BRTWLS is expected to be similar to that of Western Ghats with a significant proportion of Eastern Ghats elements as well.

It is the only large patch of forest left outside the main Western Ghats. The entire spectrum of macro habitats is represented within the $540 \mathrm{~km}^{2}$ of the reserve. The extent of the major vegetation within the reserve has also been worked out (Ramesh, 1989). Deciduous (moist and dry) comprises about 61.1 per cent, scrub 28.2 per cent, and grassland about 3.4 per cent. Higher elevation areas are characterized by evergreen (6.5\%) and high altitude sholas (0.8\%) (Ramesh, 1989).

There are several records on the avifauna of BRT WLS by Morris (1939), Ali (1942a,b; 1943a,b,c), Srinivasa et al. (1997), and Jayadeva (pers. comm.). Since Ali's report, there have been additions to the bird list by Morris (1927, 1939), Baskaran (1992) and Karthikeyan et al. (1995). We recorded further additions to the bird list as part of an ongoing project on bird and butterfly diversity in BRT Wildlife Sanctuary. 


\section{Methodology}

We have been monitoring bird diversity in and around the Sanctuary since August 1998. A series of transects were laid in every vegetation type of the Sanctuary and birds were censused over different seasons. Birds were recorded in the major vegetation types as well as in water bodies, human habitations and agro-ecosystems for 18 months. Apart from this, chance encounters were also recorded, along with the habitat in which they were observed. All identifications are based on Ali and Ripley (1983, 1995). Scientific names and taxonomic classification is after Inskipp et al. (1996).

\section{Results}

A total of 245 species of birds have been recorded in the Sanctuary till date. We have added 28 species to the existing list of 215 compiled by Srinivasa et al. (1998). This study, which covered the entire Sanctuary, recorded a total of 229 species. While the other studies were conducted in only a few areas of the Sanctuary, we covered a large area, including the three newly constructed reservoirs (in the 1970s). These reservoirs have subsequently attracted a large number of water birds. Out of 28 newly recorded species, 15 are water birds.

Apart from the area covered, we surveyed for 18 months as opposed to Srinivasa et al. (1998) who spent 10 days and Morris who surveyed on and off for most of his life. However, Morris is unlikely to have visited the northern part of the Sanctuary. Morris recorded a total of 125 species, Srinivasa et al. 194 species and Ali, 60 species. Salim Ali surveyed only a part of the Sanctuary during the bird survey of erstwhile Mysore State.

The following is the annotated checklist for the newly recorded birds of BRT Wildlife Sanctuary. A complete list of birds (246 species) of BRT is given in Table 1.

\section{Annotated checklist of the newly recorded birds}

Phalacrocoracidae

Great Cormorant (Phalacrocorax carbo): Rare. Winter visitor. Spotted a large group of 50 individuals in the Suvarnavathi Reservoir during November 1998.

Little Cormorant (Phalacrocorax niger): Common. Seen in ponds, tanks, marshes and reservoir within the Sanctuary. Seen throughout the year on several occasions.

\section{Ardeidae}

Grey Heron (Ardea cinerea): Uncommon. Seen twice, one individual each time. Seen in the southern part of the Sanctuary in November 1998.

Green-backed Heron (Butorides striatus): Rare. Two individuals seen at dusk in a pond in Biligiri Rangaswamy Hills. April 1999.
Cattle Egret (Bubulcus ibis): Common at lower elevations, where there are irrigated fields. Seen throughout the year.

Great Egret (Casmerodius albus): Rare. Only one record from the Suvarnavathi Reservoir. Seen in November 1998.

\section{Ciconiidae}

Painted Stork (Mycteria leucocephala): Rare. Seen two individuals in the Suvarnavathi Reservoir. Seen in November 1998.

White-necked Stork (Ciconia episcopus): Rare. Seen only one individual in the Suvarnavathi Reservoir. Seen in November 1998.

Threskiornithidae

Asian White Ibis (Threskiornis melanocephalus): Rare. Seen flying over the Sanctuary. Not seen feeding in any part of the Sanctuary. Seen in September 1998.

Anatidae

Bar-headed Goose (Anser indicus): Rare. Winter visitor. Seen flying over the Sanctuary. Not seen feeding in any part of the Sanctuary. Seen in October 1998.

Spot-billed Duck (Anas poecilorhyncha): Common in the reservoir, ponds and tanks of the Sanctuary. Seen during winter 1999 and 2000.

\section{Accipitridae}

Black Baza (Aviceda leuphotes): Rare. Only one individual recorded from the dry deciduous forest. Seen in November 1998.

Changeable Hawk-Eagle (Spizaetus cirrhatus): Common in the dry and moist deciduous forests of the Sanctuary.

Osprey (Pandion haliaetus): Uncommon? Winter visitor. Recorded four times during winter 1998-99.

Turnicidae

Yellow-legged Button Quail (Turnix tanki): Uncommon? Recorded twice (3 and 4 individuals respectively) in the scrub jungle of the northern part of the Sanctuary.

Jacanidae

Bronze-winged Jacana (Metpidius indicus): Common in the ponds and lakes of the Sanctuary.

Burhinidae

Stone Curlew (Burhinus oedicnemus): Rare? Two individuals seen during breeding season in the scrub jungle of the southern part of the Sanctuary. Seen in April 1999. 
Charadriidae

Common Sandpiper (Actitis hypoleucos): Uncommon. Winter visitor. Spotted only a couple of times (in a stream). Seen in December 1998.

Common Snipe (Gallinago gallinago): Uncommon. Winter visitor. Spotted 10 individuals in B.R. Hills usually in the same locality. Seen in January 1999.

Columbidae

Pompadour Green Pigeon (Treron pompadora): Common. Found feeding on these fruits of Ficus sp.

Meropidae

Blue-tailed Bee-eater (Merops philippinus): Rare. Recorded only once (2 individuals). Seen in March 1999.

Picidae

Common Flame-backed Woodpecker (Dinopium javanense): Common in the dry and moist deciduous forests.

Alaudidae

Red-winged Bush Lark (Mirafra erythroptera): Common in the drier parts of the Sanctuary. Also seen in agricultural fields.

Sturnidae

Rosy Starling (Sturnus roseus): Common. Winter visitor.

Sylviidae

Booted Warbler (Hippolais caligata): Common during winter. Seen in the dry and moist deciduous forests.

Muscicapidae

White-bellied Blue-Flycatcher (Cyornis pallipus): Uncommon. Seen in the evergreen forests at high elevations.

Ploceidae

Red Munia (Amandava amandava): Common in the fields and scrub jungle.

Black-headed Munia (Lonchura malacca): Common in the fields.

\section{Acknowledgements}

This work was funded in part by ATREE, Bangalore and CIFOR, Indonesia. We thank Drs. K.N. Ganeshaiah and R. Uma Shaanker for their encouragement. We are grateful to Dr. T. Ganesh for helpful suggestions.

\section{References}

Ali, S. (1942a). The Birds of Mysore. Part I. Journal of the Bombay Natural History Society 43(2): 130-147.

Ali, S. (1942b). The Birds of Mysore. Part II. Journal of the Bombay Natural History Society 43(3): 318-341.

Ali, S. (1943a). The Birds of Mysore. Part III. Journal of the Bombay Natural History Society 43(4): 573-595.

Ali, S. (1943b). The Birds of Mysore. Part IV. Journal of the Bombay Natural History Society 44(1): 9-26.

Ali, S. (1943c). The Birds of Mysore. Part V. Journal of the Bombay Natural History Society 44(2): 206-220.

Ali, S. and S.D. Ripley (1983). Handbook to the Birds of India and Pakistan (Compact edition). Oxford University Press and BNHS, Mumbai.

Ali, S. and S.D. Ripley (1995). The Pictorial Guide to the Birds of Indian Sub-continent. Oxford University Press and BNHS, Mumbai.

Baskaran, S.T. (1992). Sighting of Dusky Horned Owl. Newsletter for Birdwatchers 32(9+10): 17.

Inskipp, T., N. Lindsey and W. Duckworth (1996). An Annotated Checklist of the Birds of the Oriental Region. Oriental Bird Club, UK. Karthikeyan, S., J.N. Prasad and T.S. Srinivasa (1995). Yellowthroated Bulbul Pycnonotus xantholaemus (Jerdon) at Biligirirangan Hills, Karnataka. Journal of the Bombay Natural History Society 92 (1): 123-124.

Manakadan, R., J.C. Daniel, A.R. Rahmani, M. Inamdar and G. Ugra (1998). Standardized English common names of the birds of the Indian subcontinent - A proposal. Buceros 3(2): 55pp. Bombay Natural History Society, Mumbai.

Morris, R.C. (1927). A junglefowl problem. Journal of the Bombay Natural History Society 38:190.

Morris, R.C. (1939). On the occurrence of the Banded Crake (Rallus c. amauroptera) and the Malabar Woodpecker (Macropicus j. hodgsonii) in the Biligirirangan Hills, S. India. Journal of the Bombay Natural History Society 40(4): 763.

Ramesh, B.R. (1989). Flora of Biligirirangan Hills. Ph.D. Thesis, Madras University, Madras (Unpublished)

Srinivasa, T.S., S. Karthikeyan and J.N. Prasad (1997). Faunal survey of Biligiri Rangaswamy Temple Wildlife Sanctuary. Merlin Nature Club, Bangalore. 


\begin{tabular}{|c|c|c|c|c|c|c|c|c|c|c|c|c|c|}
\hline \multirow[b]{2}{*}{ Common Name } & \multirow[b]{2}{*}{ Scientific Name } & \multicolumn{4}{|c|}{ Observers } & \multirow[b]{2}{*}{ Status } & \multirow[b]{2}{*}{ Common Name } & \multirow[b]{2}{*}{ Scientific Name } & \multicolumn{4}{|c|}{ Observers } & \multirow[b]{2}{*}{ Status } \\
\hline & & SA & RM & SK & AR & & & & SA & RM & SK & AR & \\
\hline Podicipitidae & & & & & & & Hen Harrier & Circus cyaneus & & & $\checkmark$ & & $\mathrm{R}$ \\
\hline Little Grebe & Tachybaptus ruficollis & & & $\checkmark$ & $\checkmark$ & $\mathrm{C}$ & Eurasian Marsh Harrier & Circus aeruginosus & & & & $\checkmark$ & UC \\
\hline & & & & & & & Short-Toed Snake Eagle & Circaetus gallicus & & & $\checkmark$ & $\checkmark$ & UC \\
\hline Phalacrocorcidae & & & & & & & Crested Serpent Eagle & Spilornis cheela & & & $\checkmark$ & $\checkmark$ & VC \\
\hline Great Cormorant & Phalacrocorax carbo & & & & $\checkmark$ & R & Osprey & Pandion haliaetus & & & & $\checkmark$ & UC \\
\hline Little Cormorant & Phalacrocorax niger & & & & $\checkmark$ & C & & & & & & & \\
\hline Ardeidae & & & & & & & Falconidae & & & & & & \\
\hline Grey Heron & & & & & & & Peregrine Falcon & Falco peregrinus & & & $\checkmark$ & $\checkmark$ & C \\
\hline Green-backed Heron & $\begin{array}{l}\text { Ardea cinerea } \\
\text { Butorides striatus }\end{array}$ & & & & $\checkmark$ & UC & Lesser Kestrel & Falco naumanni & & $\checkmark$ & $\checkmark$ & & $\mathrm{R}$ \\
\hline Indian Pond-Heron & $\begin{array}{l}\text { Butorides strlatus } \\
\text { Ardeola grayii }\end{array}$ & & & $\checkmark$ & $\checkmark$ & $\begin{array}{l}\mathrm{R} \\
\mathrm{VC}\end{array}$ & Common Kestrel & Falco tinnunculus & $\checkmark$ & $\checkmark$ & & $\checkmark$ & C \\
\hline Cattle Egret & Bubulcus ibis & & & & $\checkmark$ & C & Phasianidae & & & & & & \\
\hline Great Egret & Casmerodius albus & & & & $\checkmark$ & $\mathrm{R}$ & Grey Francolin & Francolinus pondicerianus & & $\checkmark$ & $\checkmark$ & $\checkmark$ & $\mathrm{C}$ \\
\hline Intermediate Egret & Mesophoyx intermedia & & & $\checkmark$ & $\checkmark$ & $\mathrm{R}$ & Jungle Bush-Quail & Perdicula asiatica & & & $\checkmark$ & $\checkmark$ & $\mathrm{C}$ \\
\hline Little Egret & Egretta garzetta & & & $\checkmark$ & $\checkmark$ & VC & Painted Bush-Quail & Perdicula erythrorhyncha & $\checkmark$ & $\checkmark$ & $\checkmark$ & $\checkmark$ & UC \\
\hline & & & & & & & Red Spurfowl & Galloperdix spadicea & & $\checkmark$ & $\checkmark$ & $\checkmark$ & VC \\
\hline Ciconiidae & & & & & & & Painted Spurfowl & Galloperdix lunulata & & & $\checkmark$ & $\checkmark$ & UC \\
\hline Painted Stork & Mycteria leucocephala & & & & $\checkmark$ & UC & Grey Junglefowl & Gallus sonneratii & $\checkmark$ & $\checkmark$ & $\checkmark$ & $\checkmark$ & VC \\
\hline White-necked Stork & Ciconia episcopus & & & & $\checkmark$ & UC & Indian Peafowl & Pavo cristatus & & & & $\checkmark$ & C \\
\hline Threskiornithidae & & & & & & & Turnicidae & & & & & & \\
\hline Asian White Ibis & Threskiornis melanocephalus & & & & $\checkmark$ & UC & Yellow-legged Buttonquail & Turnix tanki & & & & $\checkmark$ & UC? \\
\hline Anatidae & & & & & & & Rallidae & & & & & & \\
\hline Bar-headed Goose & Anser indicus & & & & $\checkmark$ & $\mathrm{R}$ & Slaty-legged Crake & Rallina eurizonoides & & $\checkmark$ & & & $\mathrm{R} ?$ \\
\hline Common Teal & Anas crecca & & & $\checkmark$ & $\checkmark$ & UC & White-breasted Waterhen & Amourornis phoenicurus & & & $\checkmark$ & $\checkmark$ & $\mathrm{C}$ \\
\hline Spot-billed Duck & Anas poecilorhyncha & & & & $\checkmark$ & C & Jacanidae & & & & & & \\
\hline Dendrocygnidae & & & & & & & Bronze-winged Jacana & Metopidius indicus & & & & $\checkmark$ & C \\
\hline Lesser Whistling-Duck & Dendrocygnajavanica & & & & $\checkmark$ & $\mathrm{R}$ & & & & & & & \\
\hline Accipiteridae & & & & & $\checkmark$ & & Charadrildae & Himantopus himantopus & & & & $\checkmark$ & $U C$ \\
\hline Black-shouldered Kite & Elanus caeruleus & $\checkmark$ & & $\checkmark$ & $\checkmark$ & UC & 5 & 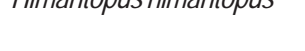 & & & & 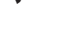 & 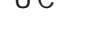 \\
\hline Black Baza & Aviceda leuphotes & & & & $\checkmark$ & $\mathrm{R}$ & Burhinidae & & & & & & \\
\hline Oriental Honey-Buzzard & Pernis ptilorhyncus & $\checkmark$ & & $\checkmark$ & $\checkmark$ & C & Stone-Curlew & Burhinus oedicnemus & & & & $\checkmark$ & UC \\
\hline Black Kite & Milvus migrans & & & $\checkmark$ & $\checkmark$ & VC & & & & & & & \\
\hline Brahminy Kite & Haliastur indus & $\checkmark$ & & $\checkmark$ & $\checkmark$ & C & Charadriidae & & & & & & \\
\hline Shikra & Accipiter badius & $\checkmark$ & & $\checkmark$ & $\checkmark$ & $\mathrm{C}$ & Red-wattled Lapwing & Vanellus indicus & & & $\checkmark$ & $\checkmark$ & $\mathrm{C}$ \\
\hline Besra Sparrow-hawk & Accipiter virgatus & $\checkmark$ & $\checkmark$ & & $\checkmark$ & C & Yellow-wattled Lapwing & V. malabaricus & & $\checkmark$ & $\checkmark$ & $\checkmark$ & UC \\
\hline Crested Hawk-Eagle & Spizaetus cirrhatus & & & & $\checkmark$ & C & Scolopacidae & & & & & & \\
\hline Rufous-bellied Hawk-Eagle & Hieraaetus kienerii & & & $\checkmark$ & $\checkmark$ & UC & Green Sandpiper & Tringa ochoropus & & $\checkmark$ & $\checkmark$ & $\checkmark$ & $\mathrm{C}$ \\
\hline Tawny Eagle & Aquila rapax & & & $\checkmark$ & $\checkmark$ & $\mathrm{R}$ & Common Sandpiper & Actitis hypoleucos & & & & $\checkmark$ & $\mathrm{C}$ \\
\hline Black Eagle & Ictinaetus malayensis & $\checkmark$ & & $\checkmark$ & $\checkmark$ & C & Pin-tailed Snipe & Gallinago stenura & & & $\checkmark$ & & UC \\
\hline Indian White-backed Vulture & Gyps bengalensis & & & $\checkmark$ & & $\mathrm{R}$ & Common Snipe & Gallinago gallinago & & & & $\checkmark$ & UC \\
\hline Egyptian Vulture & Neophron percnopterus & $\checkmark$ & & $\checkmark$ & & $\mathrm{R}$ & Eurasian Woodcock & Scolopax rusticola & & $\checkmark$ & & & $\mathrm{R}$ \\
\hline
\end{tabular}




\section{Common Name}

Laridae

Whiskered Tern

Columbidae

Pompadour Green-Pigeon Treron pompadora

Yellow-legged Green-Pigeon Treron phoenicoptera

Green Imperial-Pigeon

Duculabadia

Rock Pigeon

Nilgiri Wood-Pigeon

Eurasian Collared-Dove

Spotted Dove

Laughing Dove

Emerald Dove

Psittacidae

Rose-ringed Parakeet

Plum-headed Parakeet

Malabar Parakeet

Vernal Hanging-Parrot

\section{Cuculidae}

Pied Cuckoo

Large Hawk-Cuckoo

Indian Hawk-Cuckoo

Indian Cuckoo

Grey-bellied Cuckoo

Asian Koel

Small Green-billed Malkoha

Sirkeer Malkoha

Heirococcyx sparverioides

Centropodidae

Greater Coucal

Strigidae

Collared Scops-Ow

Dusky Eagle-Owl

Brown Fish-Owl

Jungle Owlet

Brown Hawk-Owl

Spotted Owlet

Mottled Wood-Owl

Caprimulgidae

Grey Nightjar
Ducula aenea

Columba livia

Columba elphinstonii

Streptopelia decaocto

Streptopelia chinensis

Streptopelia senegalensis

Chalcophaps indica

Psittacula krameri

Psittacula cyanocephala

Psittacula columboides

Loriculus vernalis

Clamator jacobinus

Heirococcyx varius

Cuculus micropterus

Cacomantis passerinus

Eudynamys scolopacea

Phaenicophaeus tristis

Phaenicophaeus leschenaulti

Centropus sinensis

Observers

SA M SK AR Status

$\checkmark \quad$ UC
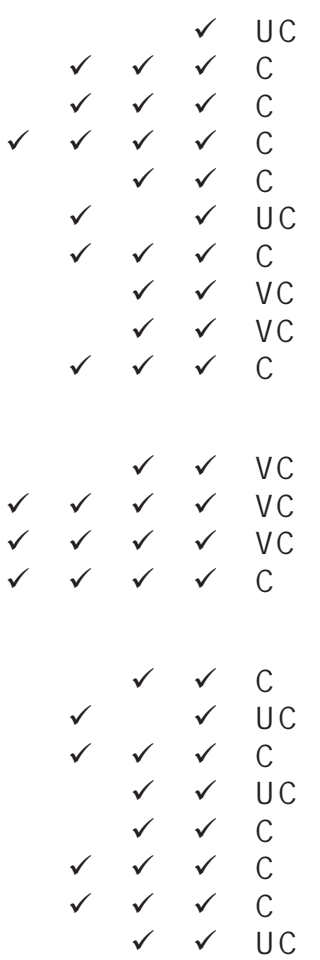

$\begin{array}{lllll}\checkmark & \checkmark & \checkmark & \checkmark & V C\end{array}$

Otus bakkamoena

Bubo coromandus

Ketupa zeylonensis

Glaucidium radiatum

Ninox scutulata

Athene brama

Strix ocellata

Caprimulgus indicus

Caprimulgus macrurus

\begin{tabular}{|c|c|c|c|c|c|c|}
\hline \multirow{2}{*}{ Common Name } & \multirow[b]{2}{*}{ Scientific Name } & \multicolumn{5}{|c|}{ Observers } \\
\hline & & SA & M & SK & AR & Status \\
\hline Indian Nightjar & Caprimulgus asiaticus & & $\checkmark$ & $\checkmark$ & $\checkmark$ & VC \\
\hline \multicolumn{7}{|l|}{ Apodidae } \\
\hline Alpine Swift & Tachymarptis melba & & $\checkmark$ & $\checkmark$ & $\checkmark$ & UC \\
\hline House Swift & Apus affinus & & & $\checkmark$ & $\checkmark$ & $\mathrm{C}$ \\
\hline Crested Tree-Swift & Hemiprocne coronata & & & $\checkmark$ & $\checkmark$ & C \\
\hline \multicolumn{7}{|l|}{ Cerylidae } \\
\hline Lesser Pied Kingfisher & Ceryle rudis & & & $\checkmark$ & $\checkmark$ & C \\
\hline \multicolumn{7}{|l|}{ Alcedinidae } \\
\hline Common Kingfisher & Alcedo atthis & & $\checkmark$ & $\checkmark$ & $\checkmark$ & C \\
\hline \multicolumn{7}{|l|}{ Dacelonidae } \\
\hline Stork-billed Kingfisher & Halcyon capensis & & & $\checkmark$ & & $\mathrm{R}$ \\
\hline White-throated Kingfisher & Halcyon smyrnensis & & $\checkmark$ & $\checkmark$ & $\checkmark$ & VC \\
\hline \multicolumn{7}{|l|}{ Meropidae } \\
\hline Chestnut-headed Bee-eater & Merops leschenaulti & $\checkmark$ & & & $\checkmark$ & UC \\
\hline Blue-tailed Bee-eater & Merops philippinus & & & & $\checkmark$ & $\mathrm{R}$ \\
\hline Green Bee-eater & Merops orientalis & $\checkmark$ & $\checkmark$ & $\checkmark$ & $\checkmark$ & C \\
\hline Blue-bearded Bee-eater & Nyctyornis athertoni & & $\checkmark$ & $\checkmark$ & $\checkmark$ & UC \\
\hline \multicolumn{7}{|l|}{ Coraciidae } \\
\hline Indian Roller & Coracias benghalensis & & $\checkmark$ & $\checkmark$ & $\checkmark$ & C \\
\hline \multicolumn{7}{|l|}{ Upupidae } \\
\hline Common Hoopoe & Upupa epops & & $\checkmark$ & $\checkmark$ & $\checkmark$ & C \\
\hline \multicolumn{7}{|l|}{ Bucerotidae } \\
\hline Indian Grey Hornbill & Ocyceros birostris & & $\checkmark$ & $\checkmark$ & $\checkmark$ & UC \\
\hline \multicolumn{7}{|l|}{ Megalaimidae } \\
\hline Brown-headed Barbet & Megalaima zeylanica & & $\checkmark$ & $\checkmark$ & $\checkmark$ & C \\
\hline White-cheeked Barbet & Megalaima viridis & $\checkmark$ & $\checkmark$ & $\checkmark$ & $\checkmark$ & VC \\
\hline Crimson-fronted Barbet & Megalaima rubricapilla & & & $\checkmark$ & $\checkmark$ & UC \\
\hline Coppersmith Barbet & M. haemacephala & & $\checkmark$ & $\checkmark$ & $\checkmark$ & C \\
\hline \multicolumn{7}{|l|}{ Picidae } \\
\hline Rufous Woodpecker & Celeus brachyurus & $\checkmark$ & & $\checkmark$ & $\checkmark$ & UC \\
\hline $\begin{array}{l}\text { Streak-throated Woodpecker } \\
\text { Lesser Yellow-naped }\end{array}$ & Picus xanthopygaeus & & $\checkmark$ & $\checkmark$ & $\checkmark$ & C \\
\hline $\begin{array}{l}\text { Woodpecker } \\
\text { Black-rumped Flame-backed }\end{array}$ & Picus chlorophus & & & $\checkmark$ & $\checkmark$ & UC \\
\hline $\begin{array}{l}\text { Woodpecker } \\
\text { Common Flame-backed }\end{array}$ & Dinopium benghalense & $\checkmark$ & $\checkmark$ & $\checkmark$ & $\checkmark$ & VC \\
\hline Woodpecker & Dinopium javanense & & & & $\checkmark$ & C \\
\hline
\end{tabular}




\begin{tabular}{ll} 
Common Name & Scientific Name \\
\hline Yellow-crowned Woodpecker & Dendrocopos maharattensis
\end{tabular}

SA RM SK AR Status

Brown-capped

Pygmy-Woodpecker

GreatFlame-backed

Dendrocopos nanus

Woodpecker

Chrysocolaptes lucidus

Pittidae

Indian Pitta

Pitta brachyura

Alaudidae

Madras Bush Lark

Mirafra affinis

Mirafra erythroptera

Ashy-crowned Sparrow-Lark Eremopterix grisea

Rufous-tailed Finch Lark Ammomanes phoenicurus

Malabar Lark Galerida malabarica

\section{Hirundinidae}

Dusky Crag-Martin

Barn Swallow

Red-rumped Swallow

Hirundo concolor

Hirundo rustica

Hirundo daurica

Laniidae

Bay-backed Shrike

Long-tailed Shrike

Lanius vittatus

Lanius schach

Brown Shrike

Lanius cristatus

\section{Sturnidae}

Chestnut-tailed Starling

Brahminy Starling

Common Myna

Jungle Myna

Hill Myna

Corvidae

Rufous Treepie

White-bellied Treepie

House Crow

Jungle Crow

Eurasian Golden Oriole

Slender-billed Oriole

Black-hooded Oriole

Black Drongo

Ashy Drongo

White-bellied Drongo

Bronzed Drongo

Spangled Drongo
Sturnus malabaricus

Sturnus pagodarum

Acridotheres tristis

Acridotheres fuscus

Gracula religiosa

Dendrocitta vagabunda

Dendrocitta leucogastra

Corvus splendens

Corvus macrorhynchos

Oriolus oriolus

Oriolus tenuirostris

Oriolus xanthornus

Dicrurus macrocercus

Dicrurus leucophaeus

Dicrurus caerulescens

Dicrurus aeneus

Dicrurus hottentottus

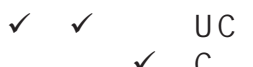

$\checkmark \checkmark \checkmark \checkmark v C$

$\checkmark \quad \checkmark \quad$ UC
Common Name

Scientific Name

SA RM SK AR Status

Greater Racket-tailed Drongo Dicrurus paradiseus

Ashy Wood-Swallow

Artamus fuscus

Bar-winged Flycatcher-shrike Hemipus picatus

Large Woodshrike

Tephrodornis gularis

Common Woodshrike

Tephrodornis pondicerianus

Large Cuckoo-shrike

Coracina macei

Coracinamelanoptera

Scarlet Minivet

Rosy Minivet

Pericrocotus flammeus

Small Minivet

White-browed Fantal

White-throated Fantail

Asian Paradise-Flycatcher

Black-naped Monarch

Pericrocotus roseus

Pericrocotus cinnamomeus

Rhipidura aureola

Rhipidura albicollis

Terpsiphone paradisi

Hypothymis azurea

Irenidae

Common lora

Gold-fronted Leafbird

Jerdon's Leafbird

Asian Fairy-Bluebird

Pycnonotidae

Red-whiskered Bulbu

Red-vented Bulbul

Yellow-throated Bulbu

White-browed Bulbul

Yellow-browed Bulbul

Black Bulbul

Sylviidae

Puff-throated Babbler

Indian Scimitar-Babbler

Tawny-bellied Babbler

Dark-fronted Babbler

Common Babbler

Rufous Babbler

Jungle Babbler

Pale-capped Babbler

Brown-cheeked Fulvetta

Common Tailor Bird

Grasshopper Warbler

Blyth's Reed-Warbler

Booted Warbler

Orphean Warbler

Lesser Whitethroat
Aegithinia tiphia

Chloropsis aurifrons

Chloropsis cochinchinensis

Irena puella

Pycnonotus jocosus

Pycnonotus cafer

Pycnonotus xantholaemus

Pycnonotus luteolus

lole indica

Hypsipetes leucocephalus

Pellorneum ruficeps

Pomatorhinus horsfieldi

Dumetia hyperythra

Rhopocichla atriceps

Turdoides caudatus

Turdoides subrufus

Turdoides striatus

Turdoides affinis

Alcippe poioicephala

Orthotomus sutorius

ocustella naevia

Acrocephalus dumetorum

Hippolais caligata

Sylvia hortensis

Sylvia curruca $\checkmark \quad \checkmark \quad \checkmark \quad r \quad C$

$\begin{array}{llllll}\checkmark & \checkmark & \checkmark & \checkmark & C\end{array}$

$\begin{array}{lllll}\checkmark & \checkmark & \checkmark & \text { UC }\end{array}$

$\begin{array}{lll}\checkmark & \checkmark & C\end{array}$

$\begin{array}{llll}\checkmark & \checkmark & \checkmark & C\end{array}$

$\begin{array}{llll}\checkmark & \checkmark & \checkmark & C\end{array}$

$\begin{array}{lllll}\checkmark & \checkmark & \checkmark & \checkmark & V C\end{array}$

$\checkmark \checkmark \checkmark \mathrm{R}^{*}$

$\begin{array}{llll}\checkmark & \checkmark & \mathrm{C} \\ \checkmark & \checkmark & \end{array}$

$\begin{array}{lll}\checkmark & \checkmark & \mathrm{R}\end{array}$

$\begin{array}{lllll}\checkmark & \checkmark & \checkmark & \mathrm{C}\end{array}$

\begin{abstract}
-
\end{abstract}

\begin{tabular}{lllll} 
& $\checkmark$ & & $\checkmark$ & \\
\hline
\end{tabular}




\section{Common Name}

Tickell's Leaf-Warble

Large-billed Leaf-Warbler

Western Crowned Warbler

Muscicapidae

Asian Brown Flycatcher

Brown-breasted Flycatcher

Rusty-tailed Flycatcher

Red-throated Flycatcher.

Black-and-Orange Flycatcher

Ficedula nigrorufa

Tickell's Blue-Flycatcher Cyornis tickelliae

Verditer Flycatcher

Nilgiri Flycatcher

Grey-headed Flycatcher

Indian Blue Robin

Oriental Magpie-Robin

Pied Bushchat

Indian Robin

Blue-headed Rock-Thrush

Blue Rock-Thrush

Malabar Whistling-Thrush

Pied Thrush

Orange-headed Thrush

Scaly Thrush

Eurasian Blackbird

Eurasian Blackbird

Cisticolidae

Zitting Cisticola

Grey-breasted Prinia

Plain Prinia

Ashy Prinia

Jungle Prinia

Paridae

Great Tit

Pied Tit

Black-lored Yellow Tit

Parus nuchalis

Parus xanthogenys

Sittidae

Chestnut-bellied Nuthatch

Velvet-fronted Nuthatch

Sitta castanea

Sitta frontalis

ciniabrunnea

Monticola solitarius

Zoothera wardii

Zoothera dauma

Cisticola juncidis

Prinia hodgsonii

Prinia socialis

Prinia sylvatica
Observers

SA RM SK AR Status

$\begin{array}{llll}\checkmark & \checkmark & \checkmark & \text { UC }\end{array}$

$\begin{array}{llllll}\checkmark & \checkmark & \checkmark & \checkmark & C\end{array}$

$\begin{array}{lllll}\checkmark & \checkmark & \checkmark & \checkmark & C\end{array}$

$\begin{array}{llll}\checkmark & \checkmark & \checkmark & \mathrm{C}\end{array}$

$\checkmark \quad \checkmark \quad \mathrm{R}$

$\begin{array}{llll}\checkmark & \checkmark & \text { UC }\end{array}$

$\checkmark \quad \mathrm{R}$

$\checkmark \quad \checkmark \quad$ VR

$\checkmark \begin{array}{lllll}\checkmark & \checkmark & \checkmark & \checkmark & \mathrm{C}\end{array}$

$\begin{array}{lll}\checkmark & \checkmark & \text { UC } \\ \checkmark & \checkmark & \text { UC }\end{array}$

$\begin{array}{llll}\checkmark & \checkmark & \checkmark & U C\end{array}$

Eumyias albicaudata

Muscicapa ceylonensis

Saxicoloides fulicata

Monticola cinclorhynchus

Myiophonus horsfieldii

Zurdus merula nigropeleus

Turdus merula similimus

$\begin{array}{lllll}\checkmark & \checkmark & \checkmark & \checkmark & C\end{array}$

$\begin{array}{lllll}\checkmark & \checkmark & \cup C\end{array}$

$\begin{array}{llll}\checkmark & \checkmark & \checkmark & V C\end{array}$

$\begin{array}{llll}\checkmark & \checkmark & \checkmark & C\end{array}$

$\begin{array}{lllll}\checkmark & \checkmark & \checkmark & \text { VC }\end{array}$

$\begin{array}{llllll} & \checkmark & \checkmark & \checkmark & \checkmark & C\end{array}$

$\begin{array}{lllll}\checkmark & \checkmark & \checkmark & \checkmark & \text { UC }\end{array}$

$\begin{array}{lllll}\checkmark & \checkmark & \checkmark & \checkmark & \mathrm{C}\end{array}$

$\checkmark \quad \mathrm{R}$

$\checkmark \quad \checkmark \quad C$

$\checkmark \begin{array}{llll}\checkmark & & & \mathrm{R} \\ & \checkmark & \checkmark & \mathrm{C}\end{array}$

$\checkmark$ R

$\checkmark \checkmark \checkmark \quad$ UC

$\begin{array}{llll}\checkmark & \checkmark & \checkmark & C\end{array}$

$\checkmark \quad \checkmark \quad C$

$\begin{array}{llll}\checkmark & \checkmark & \checkmark & C\end{array}$

$\checkmark \quad \checkmark \quad C$

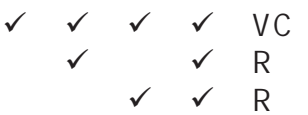

$\begin{array}{lllll}\checkmark & \checkmark & \checkmark & \checkmark & C\end{array}$

$\checkmark \quad \checkmark \quad \checkmark \quad V C$
Observers

Common Name

Scientific Name

SA RM SK AR Status

Tree Pipit Anthus trivialis

Paddyfield Pipit Anthus rufulus

Long-billed Pipit

Anthus similis

Dendronanthus indica

restwagtal

Yellow Wagtail

Grey Wagtail

Motacilla flava

Motacilla cinerea

arge Pied Wagtail

Motacilla maderaspatensis

Passer domesticus

Chestnut-shouldered Petronia Petroniaxanthocollis

Ploceus philippinus

Red Munia Amandava amandava

Plain Munia

White-rumped Munia

Hill Munia

Scaly-breasted Munia

Black-headed Munia

Lonchuramalabarica

Lonchura striata

Lonchura kelaarti

Lonchura punctulata

Lonchura malacca

Nectariniidae

Thick-billed Flowerpecker Dicaeum agile

Tickell's Flowerpecker

Dicaeum erythrorhynchos

Plain-coloured Flowerpecker Dicaeum concolor

Purple-rumped Sunbird Nectarinia zeylonica

Small Sunbird

Nectarinia minima

Nectarinia lotenia

Nectarinia asiatica

Purple Sunbird

Zosteropidae

Oriental White-Eye

Zosterops palpebrosa

Fringillidae

Common Rosefinch

Carpodacus erythrinus

Total species recorded

SA- Salim Ali; RM- R.C. Morris; SK- T. Srinivasa, S. Karthikeyan, J.N. Prasad;

AR- N.A. Aravind, Dinesh Rao, P.S. Madhusudan

R- Rare; UC- Uncommon; C- Common; VC- Very common

* - Seen by Uttangi, 2000

Common names used in this table follow Manakadan et al. (1998)

Scientific names are after Inskipp et al. (1996).

$\checkmark$ R

$\begin{array}{lllll}\checkmark & \checkmark & \checkmark & C\end{array}$ $\checkmark$

$\checkmark \quad \checkmark \quad \checkmark \quad \cup C$

$\begin{array}{llll}\checkmark & \checkmark & \checkmark & C\end{array}$

$\begin{array}{lll}\checkmark & \checkmark & V C\end{array}$

$\begin{array}{lllll}\checkmark & \checkmark & \checkmark & C\end{array}$

$\begin{array}{llll}\checkmark & \checkmark & U C\end{array}$

$\checkmark \quad \checkmark \quad C$

$\checkmark \quad C$

$\checkmark \checkmark \checkmark C$

$\begin{array}{llll}\checkmark & \checkmark & \checkmark & V C\end{array}$

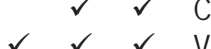

$\begin{array}{lll}\checkmark & \checkmark & \mathrm{VC}\end{array}$

$\begin{array}{llll}\checkmark & \checkmark & \checkmark & \mathrm{R}\end{array}$

$\begin{array}{lllll}\checkmark & \checkmark & \checkmark & \text { UC } \\ \checkmark & \checkmark & \checkmark & \text { VC }\end{array}$

$\checkmark \checkmark \checkmark$

$\checkmark \checkmark \checkmark \checkmark c$

$\begin{array}{llll}60 & 125 & 194 & 229\end{array}$ 